Türkiye Jeoloji Bülteni
Geological Bulletin of Turkey
$60(2017) 489-506$
doi:10.25288/tjb.360609

\title{
Termal-Asistan Optik Uyarmalı Lüminesans (TA-OSL) Tekniğiyle Milyon Yıl Mertebesindeki Jeolojik Örneklerin Tarihlendirilmesi: Gediz Taraçaları, Kula/Manisa
}

Dating of geological samples over millions of years by Thermally Assisted Optically Stimulated Luminescence (TA-OSL) technique: Gediz River Terraces, Kula/Manisa

\author{
Eren Şahiner $^{1} \mathbb{D}$, Mehmet Korhan Erturaç $\mathbb{D}^{\mathbb{D}}$, Niyazi Meriç ${ }^{1} \mathbb{D}$ \\ ${ }^{1}$ Ankara Üniversitesi, Nükleer Bilimler Enstitüsü, Lüminesans Tarihlendirme Araştırma Laboratuvarl, \\ 06100 Beşevler, Ankara \\ ${ }^{2}$ Sakarya Üniversitesi, Fen Edebiyat Fak., Coğrafya Bölümü, 54187 Serdivan/Sakarya
}

Geliş/Received : 11.11.2017 • Düzeltilmiş Metin Geliş/Revised Manuscript Received : 20.11.2017 • Kabul/Accepted : 22.11.2017 • Bask1/Printed : 29.12 .2017 Araştırma Makalesi/Research Article Türkije Jeol. Bül. / Geol. Bull. Turkey

Öz: Kuvaterner Devri ( 2.6 milyon yıl) üzerine yoğunlaşan çalışmalar, yeryüzünün yakın dönem jeolojik geçmişini, özellikle iklim değişiklikleri, tektonik ve volkanik aktivitenin canlılar ile etkileşimini ilişkilendirerek araştırmaktadır. $\mathrm{Bu}$ tarz çalışmalar, günümüzde arkeoloji, jeoloji, paleoantropoloji, coğrafya, paleosismoloji vb. farklı bilim dallarınca, disiplinler arası işbirliği ile sürdürülmektedir. Bu anlamda yerin geçmişini araştıran bu bilim dallarının, "neden", "nasıl”, "nerede" sorularının yanı sıra özellikle "ne zaman" sorularının cevabını araştırmak için en çok faydalandıkları temel bilimler, fizik ve kimya' dır. Çünkü temel bilimlerin katkısıyla yer süreçleri sonucu gerçekleşen çeşitli ölçekte tekil olayların gerçekleşme zamanlarının ve bununla birlikte bu süreçlerinin hızının ölçülebilmesi amacıyla farklı tarihlendirme teknikleri geliştirilmiş̧ir ve bu tekniklerin, güncel bilgiler ışığında, hassaslıklarının arttırılması ve uygulama sahalarının genişletilmesi konusunda araştırmalar hızla devam etmektedir.

Bu çalışmaların temel veri kaynaklarından olan inorganik çökellerin depolanma kronolojisi, önemli ölçüde, Optik Uyarmalı Lüminesans (OSL) ve Termolüminesans (TL) yaş tayini çalışmalarıyla, birkaç yüz bin yıla kadar ulaşan zaman için, desteklenmektedir. Bununla birlikte kristal yapılarındaki lüminesans oluşum mekanizması ve tuzak-yük yapısını inceleyen yeni gelişmeler kullanılarak lüminesans tarihlendirme yöntemlerinin milyon yıllar mertebesindeki yaş aralığını kapsamak adına oldukça iyi bir potansiyele sahip olduğu gösterilmektedir.

Bu çalışmada, Manisa Kula Volkanik Bölgesi içerisinde, aktivite kronolojisi çok iyi bilinen bir lav akıntısı tarafından örtülen ve milyon yıl mertebesinde depolanma tarihçesine sahip çökel katmanların yaş tayinlerinin, çeşitli lüminesans teknikler ile karşılaştırmalı olarak analizi konu edilmektedir. Bu amaçla lüminesans tarihlendirme analizlerinde yaygın olarak kullanılan doğal minerallerin sıradan tuzaklarına göre daha derinde olan tuzakları, Termal asistan OSL (TA-OSL) tekniği ile araştırılarak tarihlendirmesi literatürde ilk defa gerçekleştirilmiştir. Elde edilen sonuç (1066 \pm 90 binyıl), radyometrik tarihlendirme ile önerilen $1200 \pm 22$ binyıl yaş ile $88 \pm 10 \%$ oranında uyumludur.

Anahtar Kelimeler: Çok derin tuzaklar (VDT), Gediz taraçaları, Kula Volkanizması, Lüminesans tarihlendirme, Termal-Asistan Optik Uyarmalı Lüminesans (TA-OSL),yaş limitleri

\begin{abstract}
Studies focusing on the Quaternary, the last period of the Earth's history ( $2.6 \mathrm{Ma}$ ) is involved with interaction of biosphere with significant earth processes such as climate changes, tectonic and volcanic activities. These studies are interdisciplinary in nature, which combines questions, techniques and methodologies of archeology, geology, paleoanthropology, geography and paleoseismology. In order to understand the main questions, such as "why", "where" and "how" in earth processes, we consult physics and chemistry to answer the question "when".


Fundamental sciences provide independent dating techniques to determine the exact timing and also rate of significant events. These techniques continuously develop by means of accuracy, integrity, sensitivity and also field of application.

One of the basic sources of information of these studies, inorganic sediments, are successfully dated by Optically Stimulated Luminescence (OSL) and Thermoluminescence (TL) methods for the Late Quaternary time interval (100$200 \mathrm{ka}$ ). Recent developments in luminescence technique involves the study of formation of luminescence signal and nature of trap-charge structures in crystal lattice reveal possibility of dating older sediments deposited over million years ago.

In this study, we investigated the luminescence age of a high fluvial terrace in Kula (Manisa) Volcanic Field which is previously dated by constraining its deposition age with the radiometric dates of overlying lava flow ( 1200 Ma) by employing Thermally Assisted (TA) OSL protocol. TA-OSL stimulates deeper traps than the ordinary OSL methods extending the age limit significantly. The result of TA-OSL age $(1066 \pm 90 \mathrm{ka})$ coincides $(88 \pm 10 \%)$ with the mean of radiometric dates (1200 $\pm 22 \mathrm{ka}$ ), showing a promising success of a developing technique.

Keywords: Age limits, Gediz Terraces, Kula Volcanism, Luminescence Dating, TA-OSL, Very Deep Traps (VDT)

\section{GíRiș}

Lüminesans araştırmaları, malzeme karakterizasyonu, iyonize radyasyon dozimetrisi, kaza dozimetrisi, jeokronoloji, arkeolojik örneklerin sahtecilik testi vb. birçok araştırma alanında kritik rol oynamaktadır (McKeever, 1985; Boter-Jensen vd. 2003). TL (Termolüminesans) ve OSL (Optik Uyarmalı Lüminesans) yöntemleri bu araştırmalar arasında oldukça önemli bir yere sahiptir. 1970'lerde temelleri atılan bu yaş tayini tekniği, günümüze kadar ivmeli bir şekilde gelişme göstererek inorganik malzemelerin yaş tayini çalışmalarında anahtar rol oynayan bir teknik haline gelmeyi başarmıştır. Geç Kuvaterner (son 100-200 bin yıl) içerisinde depolanan çökel katmanların en son 1şığa maruz kaldığı (gömülme) tarih ve arkeolojik teknoloji ürünlerinin (çanak, çömlek, seramik, briket, harç vb.) üretim tarihi bu teknikler aracılığıyla hesaplanabilir. Özellikle son yıllarda geliştirilen SAR (Single Aliquot Regenerative Dose; Wintle ve Murray, 2000) gibi protokollerle \% 3-10 hata ile yaş tayini yapılabilen teknik, radyokarbon ve radyoizotop yaş tayini yöntemleri ile birlikte yaygın kullanıma girerek yer bilimleri literatüründe kabul edilmiştir.

Lüminesans tarihlendirme ve retrospektif dozimetri; kuvars, feldspat vb. doğal minerallerin iyonize radyasyonu soğuran birer dozimetri gibi davranarak birim zamanda birim kütle başına soğurduğu enerjinin ölçülmesi temeline dayanır. Doğadaki sabit düşük seviyeli iyonize radyasyonun kaynağı uzay merkezli kozmik radyasyon ve ${ }^{232} \mathrm{Th},{ }^{40} \mathrm{~K},{ }^{87} \mathrm{Rb}$, doğal $\mathrm{U}$ serilerinin bozunumunda yayınlanan dalga veya parçacık halindeki enerjidir. Bu radyasyon dozu kuvars, feldspat vb. doğal minerallerin kristal yapısı içerisindeki elektron ve hol tuzaklarında elektron ve hollerin birikmesine neden olur ve dişarıdan bir etki gelmediği sürece kararlı olarak zamanın bir fonksiyonu olarak birikmeye devam eder. $\mathrm{Bu}$ durum ancak dişarıdan 1S1, 1ş1k, mekanik vb., gibi bir etki geldiğinde bozulur. Bu etkilere doğal süreçlerden örnek olarak ayrışma, aşınma ve taşınma süreçleri ile 1şığa maruz kalma ya da deprem, volkanik aktivite gibi 1sı açığa çıkaran olaylar örnek verilebilir. Bu etkiler sonrası kristal yapısı içerisindeki tüm tuzaklar hızla boşalır ve böylece lüminesans saati sıfırlanmış olur. Gömülme sonrası tuzakların yeniden sabit bir hızla dolmaya başlamasıyla lüminesans saatin yeniden çalıştığ varsayılmaktadır. Tarihlendirilmesi istenen çökel katmanı ya da arkeolojik buluntunun, yerinde, 1ş1k görmeden örneklenmesi, hedef minerallerin laboratuvar ortamında güvenli 1şık koşullarında ayıklanması, kristal yapısı içerisindeki tuzakların yapısının ve yükünün kontrollü olarak araştırılması ile en son ısıya ve 1şığa maruz kalınan zamana 
geri dönülebilir. Laboratuvar ortamında kontrollü uyarma işlemi 1sıyla yapılırsa; termolüminesans (TL), 1şıkla yapılırsa; optik uyarmalı lüminesans (OSL) tekniği olarak isimlendirilir (Aitken, 1985).

Kristal yapının daha iyi anlaşılmasına dair yapılan güncel çalışmalar, kristallerin bant yapısı hakkında daha detaylı bilgiler elde etmemize neden olmuştur. Bu sayede tuzak-yük yapısı, tuzaklanmış elektronların yeniden birleşmesi ve tuzak derinliği hakkında önemli bilgiler üretilmiştir. Lüminesans tarihlendirmenin temeli olan kuvars ve feldspat vb. minerallerin bant yapısı içinde daha derinde daha kararlı tuzaklara sahip olduğu gösterilmiştir. $\mathrm{Bu}$ tuzaklardan elde edilecek çıktılar, arkeolojik/ jeolojik yaş limitinin arttırılması ve yüksek doz dozimetrisi gibi konu hakkında yeni araştırmalara yol açacağı gibi mevcut rutin lüminesans tarihlendirme çalışmalarında da örnek hazırlama sürecini basitleştirecek son derece kritik bilgilere ulaşılmasını mümkün kılacaktır.

Kuvars mineralinin OSL sinyalinin hızlı bileşen kısmından (ilk 1-5 sn) yapılabilen tarihlendirme çalışmaları, OSL doz-cevabın doyuma ulaşmasından dolayı yaklaşık 100-200 binyıl civarında sınırlanmaktadır. Bununla birlikte güncel çalışmalarda yapılan deneylerde kuvars mineralinin $200-300^{\circ} \mathrm{C}$ 'de 1 sitıldığında TermalTransfer (TT-OSL) sinyali olduğu gözlenmiştir. $\mathrm{Bu}$ sinyalin doyumunun daha yüksek dozlarda olduğu ve 1 milyon yıla kadar yaş tayini yapılabileceği, lüminesans özellikleri ile birlikte Wang vd. (2006a, b) tarafından gösterilmiştir. Ayrıca Wang vd. (2007) tarafından lös depolarının SAR tekniği ile başarılı bir şekilde 780 binyıl yıla tarihlendirilmiştir. Jacobs vd. (2011) Güney Afrika Cape sahillerine ait denizel örneklerin 400 binyıla kadar tarihlendirmiştir.

Yaş limitlerini arttırmaya yönelik diğer çalışmalar da mor (violet) 1şık ile uyarma üzerine yoğunlaşmıştır. Mor 1şık mavi 1şığa göre daha derindeki tuzakları uyarabileceği için daha ölçülebilen dozlimitlerini arttırabilir(Ankjærgaard vd. 2013, 2015, 2016). Ankjærgaard vd. (2016), Çin lös sekansından elde edilmiş örneklerden ayıklanan kuvars mineralinde, uyarım 1şı̆̆ı olarak maviden daha enerjik olan mor 1 şı ̆̆ kullanarak, 600 bin yıla ulaşan yaş tayin etmişlerdir.

Bahsi geçen tekniklerin uygulama sahası ve fizik prensipleri üzerine tartışmalar halen devam ederken yukarıda belirtildiği şekilde bazı başarılı uygulamaları bulunmaktadır. $\mathrm{Bu}$ çalışmada kullanmış olduğumuz Termal Asistan (Is1Destekli, TA) OSL tekniği de sıradan lüminesans tuzaklarında göre daha derindeki tuzaklardan eşdeğer doz değerlendirmesi yapabilen oldukça önemli bir tekniktir.

$\mathrm{Bu}$ araştırmada volkanik bir kayaç ile örtülen akarsu çökelinden alınan yaşlı (standart lüminesans tekniklerine göre) çökel örneğinin TAOSL yöntemi ile tarihlendirilmesi yapılmıştır. $\mathrm{Bu}$ kapsamda çalışmayla gerçekleştirilecek çıktılar hem jeolojik hem de metodolojik açıdan önem arz etmektedir. TA-OSL tekniği ile lüminesans tarihlendirme yaş sınırlarının milyon yıllar mertebesine çıkarılabileceği gösterilmiştir.

\section{INCELEME ALANI}

İnceleme alan1, Bat1 Anadolu'da Manisa İl sınırları içerisinde yer alan Kula Volkanik Bölgesinde (KVB) yer almaktadır (Şekil 1). Bu bölgede Orta-Geç Kuvaterner içerisinde üç farklı evrede (sırasıyla Burgaz, Elekçitepe ve Divlittepe volkanikleri) gelişen bazaltik volkanizmaya ait ürünler (lav akıntıları, skorya konileri vb.) yaygın olarak gözlenir (Ercan vd., 1983; Ercan, 1993; Şen vd., 2017; Şekil 1B). Kula Volkanizması, geçmişten günümüze yürütülen çalışmalarla jeolojik özellikleri (Ercan, 1983; RichardsonBanbury, 1996), özellikle de son dönemde yapılan çalışmalarla üretilen radyometrik tarih ve paleomanyetik ölçümler ile aktivite kronolojisi (Borsi vd., 1972; Richardson-Banbury, 1996; Westaway vd., 2003, 2004; Maddy vd., 2005, 
2012a, 2012b, 2015, 2017; Heineke vd., 2016) en iyi bilinen volkanik bölgelerimizden birisidir. KVB'nin doğu kesiminde, ilk volkanik evreye (Burgaz Volkanitleri, $\beta 2$ ) ait lav akıntıları belirgin bir plato morfolojisi sunar (Şekil 1B ve Şekil 2; Erinç, 1970). bu seviyeler, en alt taraçanın (T-1) kanal çakılları içerisinde bulunan bir taş alet nedeniyle ayrı bir öneme sahiptir (Maddy vd., 2015). Taraçaların tarihlendirilmesi, seviyelerin konumları ve taraça çökellerinin radyometrik (Ar/Ar) yaş tayinleri ile gerçekleştirilmiştir (Maddy vd. 2015 ve 2017).
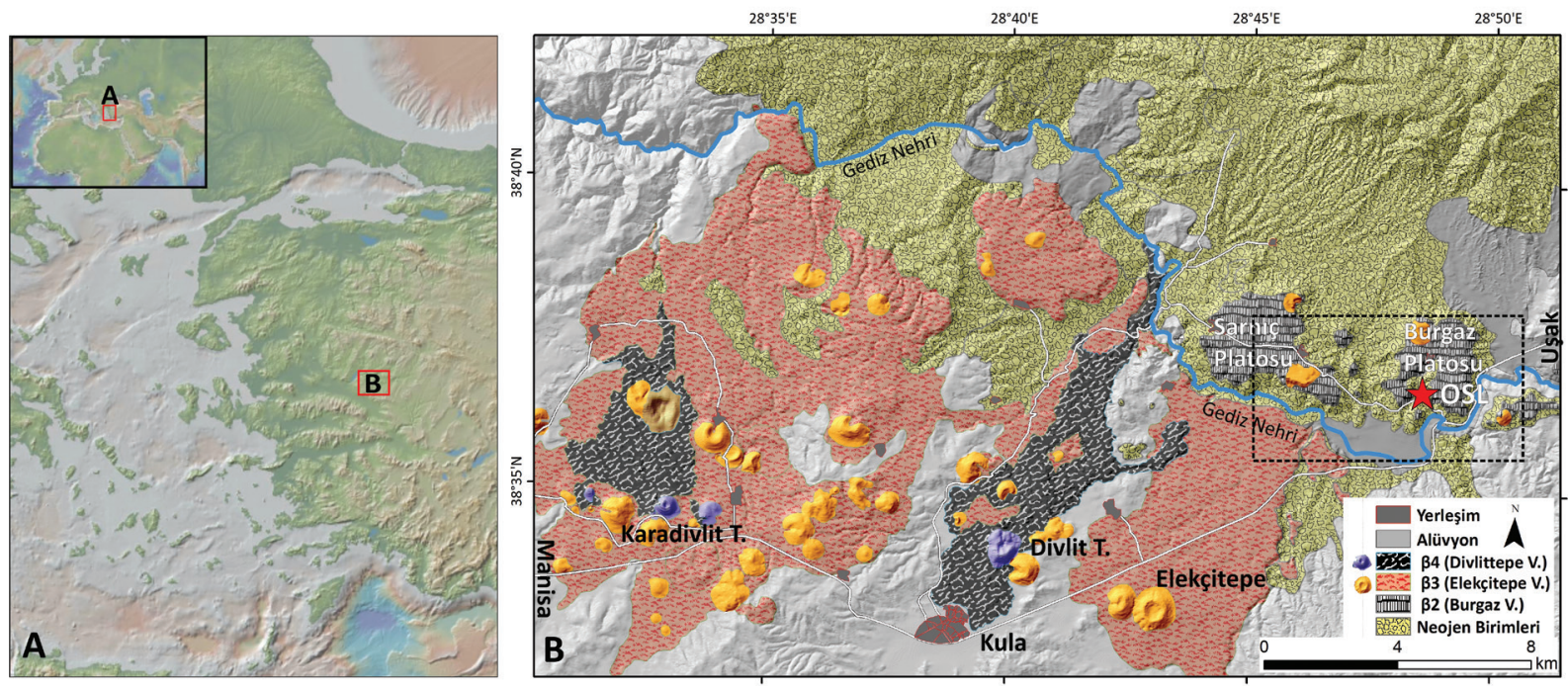

Şekil 1. A. İnceleme alanının batı Anadolu'daki konumu (Geomapapp) B. Kula Volkanik Bölgesi’nin orta ve doğu kesiminin Neojen-Kuvaterner jeoloji haritası (Şen vd., 2017'den düzenlenerek), çalışma alanı KVB'nin doğu sınırında yer alan Burgaz Platosu GB sinırında yer almaktadır.

Figure 1. A Location map of the study area in western Anatolia, B. Quaternary Geological map of the western portion of the KVF (modified after Şen et al., 2017) detailing the position of Burgaz basalt plateau and sample site.

Yakın dönemde, bu evreye ait lav akıntıları tarafından örtülen Gediz Nehri'ne ait taraça sistematiği kapsamlı olarak çalışılmıştır (detaylı bir derleme için: Maddy vd., 2017). İlk olarak Westaway vd. (2003 ve 2004) tarafindan tanımlanan ve Batı Anadolu'nun Kuvaterner boyunca tektonik gelişimi (Maddy vd., 2005, 2012) ve drenaj sisteminin evriminin (Veldkamp vd., 2015) anlaşılması için anahtar özelliği olan
Buna göre sistematiğin 11 ayrı basamak olarak, denizel izotop katları (MIS) 52 ile 38 arasında geliştiği önerilmektedir (Maddy vd., 2017). Buna göre sistematik olarak en genç taraça (T-1), Burgaz Platosunun güney yamacinda, Miyosen yaşlı Ahmetler formasyonu (Ersoy vd., 2010) ve Burgaz Volkanitleri ile sınırlanmaktadır (Şekil 3). 


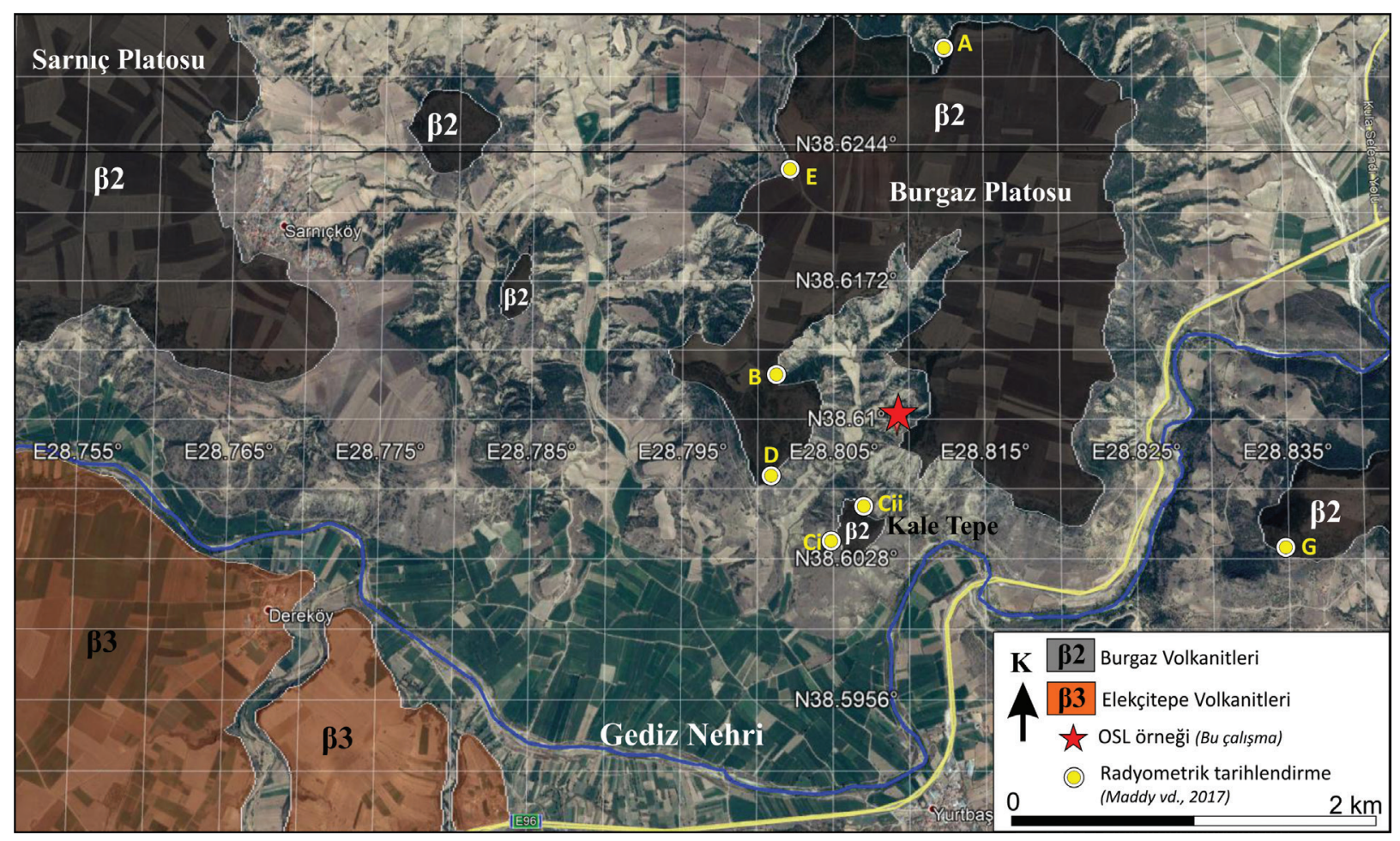

Şekil 2. Çalışma alanının Google Earth $^{\mathrm{TM}}$ görüntüsü üzerinde görünümü. Sarı daireler Maddy vd. (2017) de değerlendirilen radyometrik tarihlendirme örneklerinin, kırmızı yıldız ise OSL örneğinin konumunu göstermektedir.

Figure 2. Detailed map of the study area over Google Earth ${ }^{T M}$ image. Yellow circles show the position of radiometric ages and red star shows the location of OSL sample

Bu çalışma için elde edilen OSL örneği, geç Miyosen yaşlı karasal Ahmetler formasyonuna ait haki renkli killi seviye üzerine uyumsuz olarak gelen ve belirgin bir akarsu deposu niteliği sunan taraça seviyesi içerisinden alınmıştır (Şekil 2 ve Şekil 3). Toplam kalınlığı 3.5 metre olan bu taraça seviyesi, altta 1 metre kalınlığında kaba kanal çakıl ve blokları ile başlar (I). Bu seviyeyi $\sim 60 \mathrm{~cm}$ kalınlığında yer yer çapraz tabakalı ve tekne yapılı siltli ince kum seviyesi izler (II). Üste doğru $1 \mathrm{~m}$ kalınlığında taşkın ovası siltleri bulunmaktadır (III). Taraça kesiti, üst kısmında 1 m kalınlığında yaygı akıntısı silt ve çakılları ile paleosol seviyesi (IV) ile sonlanır. Bu seviye, Kula Volkanizması'nın ilk evresine (Burgaz Volkanitleri) ait bazaltik kül ile bloklar ve sonrasında da masif lav akıntısı ile örtülmektedir (Şekil 3B). Örnek, taraça tabanında yer alan kanal çakıllarının üzerinde yer alan $\sim 60$ cm kalınlığındaki siltli ince kum seviyesinde siyah PVC tüp çakılarak alınmıştır. Örneğinin deniz seviyesinden yüksekliği 542.5 metre, günümüz yüzeyden derinliği ise $7.5 \mathrm{~m}$ olarak ölçülmüsşür. $\mathrm{Bu}$ seviye, konumu ve yüksekliği göz önünde bulundurularak, Maddy vd. (2015 ve 2017) Gediz taraça sistematiği sınıflandırmasına göre T-1 olarak değerlendirilmiştir. Kula bölgesinde yer alan Elekçitepe ( 250 binyıl) ve Divlittepe (4010 binyıl) fazlarına ait lav akıntıları önce eğim yönünde sonra da Gediz Nehri vadisi içerisinde akmaktadır. Bu nedenle günümüz Gediz Nehri ana kanalı, KVB içerisinde kuzeye doğru büklüm yapmakta ve yatağını volkanizma ile eş zamanlı terketmektedir (Ozaner, 1992; Gorp vd., 2014). Buna göre taraçaların çökelme yaşı, örten bazalt akıntısı ile sınırlandırılabilir. Bundan hareketle, T1 seviyesinin Burgaz Volkanitlerinin Ar/Ar 
tarih analizleri sonucu MIS38 içerisinde ( 1280 binyıl) depolandığ 1 önerilmiştir (Maddy vd., 2017). Gediz Nehri'nin güncel taşkın ovasından $\sim 150$ m yukarıda bulunan bu çökeller $\sim 0.16 \mathrm{~mm} /$ yıl bölgesel yükselim hızı hesaplanmasına olanak sağlamaktadır (Maddy vd., 2005).

$\mathrm{Bu}$ çalışmada temel alınan hipotez, Gediz Nehri taraça çökellerinin literatürde de önerildiği gibi volkanizma ile eş yaşlı olduğu yönündedir. $\mathrm{Bu}$ hipotez, TA-OSL tarihlendirme yöntemi ile test edilmiştir.

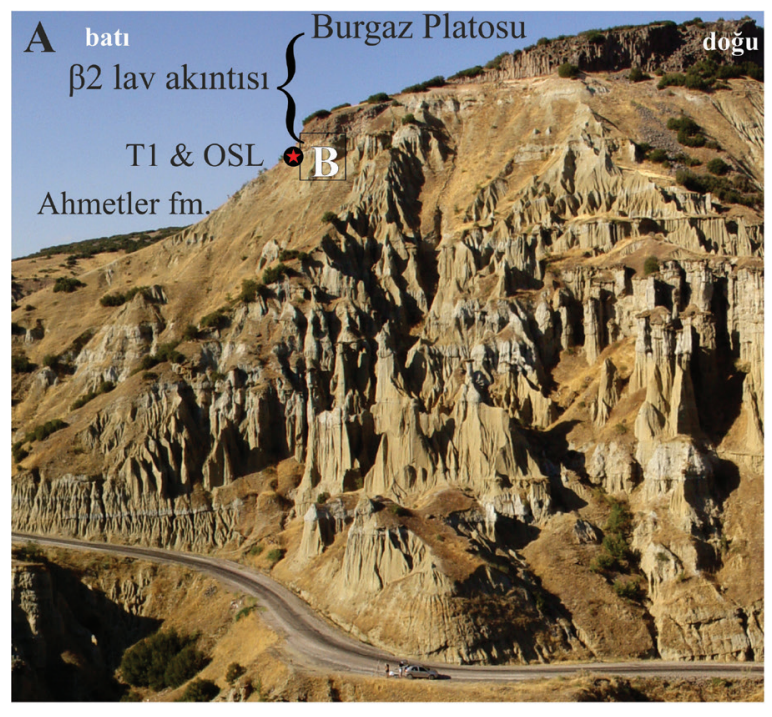

uyarmanın yollarından biri $500^{\circ} \mathrm{C}$ 'nin üstündeki sicaklıklarda okuma yapmaktır ancak, yüksek sıcaklıklara çıkıldıkça örnek akkor haline gelmeye başlayacak ve siyah cisim 1şıması lüminesansdan daha baskın hale gelecektir. Ayrıca okuma için geliştirilen günümüz cihazlarının okuyabildiği maksimum sicaklıklar $500-700^{\circ} \mathrm{C}$ 'dir. $\mathrm{Bu}$ tuzaklara ulaşmanın yollarından biri Termal Asistan OSL (TA-OSL) tekniğidir. Bu teknik TL ve OSL tekniğinin bir kombinasyonudur. Örnek önce uygun sıcaklığa kadar ısıtılır ve bu sicaklıkta

Şekil 3. A. Ahmetler formasyonu ile Burgaz Volkanitlerine ait lav akıntıları arasında sınırlanan Gediz taraça kesitinin (T1) A. genel konumu B. ölçülü taraça kesiti üzerinde laminalı siltli ince kum fasiyesinden alınan OSL örneğinin konumu, ölçek $1 \mathrm{~m}$ uzunluğundadır.

Figure 3. (A) Position of Gediz River Terrace section in between Burgaz Volcanics and Ahmetler formation (B) measured section of the terrace layer, facies and the position of OSL sample (scale is $1 \mathrm{~m}$ long)

\section{MATERYAL VE YÖNTEM}

\section{Çok Derin Tuzaklar (VDT)}

Çoğu TL, OSL fosfor malzemeleri geniş bant aralığına $(1-12 \mathrm{eV})$ ve yukarıda bahsedilen tuzakların dışında daha derin enerji seviyelerinde tuzaklara sahiptirler (Kittel, 2004). Bunlara çok derin tuzaklar (Very Deep Traps, VDT) denir ve ancak $500^{\circ} \mathrm{C}$ 'nin üzerindeki sicaklıklarda uyarılabilirler (Şahiner, 2015). Bu tuzakları
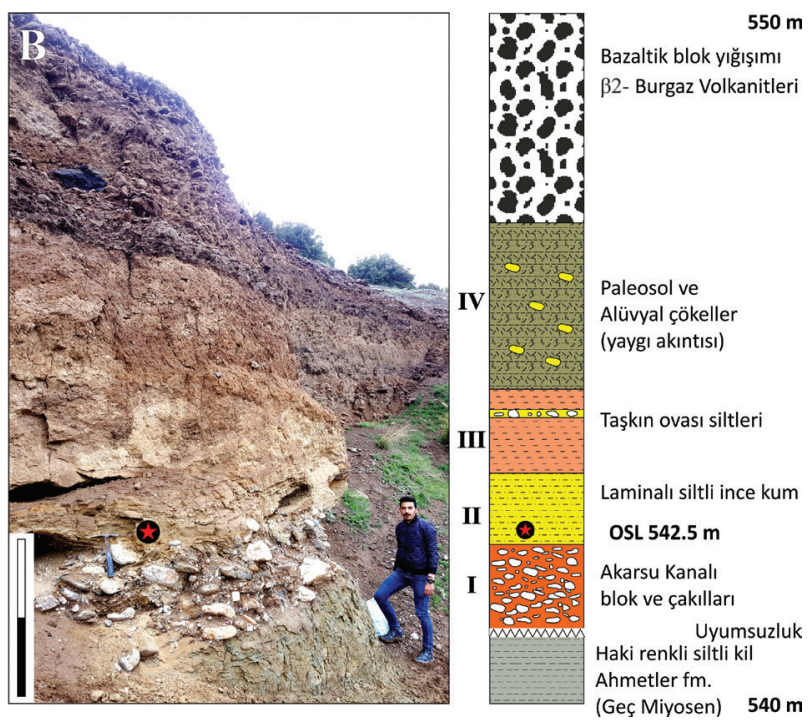

iken 1şıkla uyarma yapılır. Derin tuzaklarda kullanabilmek için önce $500^{\circ} \mathrm{C}^{\prime}$ ye kadar bir TL okumas1 yap1lır (Şahiner, 2015). Bu sicaklığa kadar olan tuzakların sıfırlandığı gözlendikten sonra örnek uygun sicaklikta 1sit1lır ve bu sıcaklıkta iken OSL okuması yapılır. Literatürde $\mathrm{CaF}_{2}: \mathrm{N}, \mathrm{Al}_{2} \mathrm{O}_{3}: \mathrm{C}$, kuvars, feldspat, polimineral ve apatit gibi malzemeler bu teknikle incelenmiştir (Bulur ve Göksu, 1999; Kitis vd., 2010; Polymeris vd., 2010; Polymeris ve Kitis, 2012; Kitis vd., 
2013; Polymeris vd., 2015 a, b; Şahiner, 2015). $\mathrm{Bu}$ tür derin tuzakların radyasyon dozimetrisi ve yaş tayininde kullanımının bazı avantajları bulunmaktadır. Bu tuzaklar kendilerinden daha sığ olan tuzaklara göre daha kararlı ve daha uzun ömre sahiptir (Şahiner, 2015). Bu özellikler TL ve OSL uygulamaları açısından çok büyük öneme sahiptir. Bu şekilde daha hassas ve geniş doz aralığında eşdeğer doz tespiti yapılabilir ve dolayısıyla tarihöncesi arkeoloji ve Kuvaterner jeolojisi uygulamalarında daha geniş aralıkta ve hassasiyetle yaş hesabı yapılabilir (Şahiner, 2015).

\section{TA-OSL Protokolü}

Kula volkanik bölgesinde, Gediz Nehri yüksek taraça kesitinden lüminesans tarihlendirmeye uygun olarak 1şık geçirmez karotlar yardımıyla alınan numune, zayıflatılmış kırmızı (güvenli) 1şık altında uygun laboratuvar koşullarında karanlık odada hazırlanmıştır. Buna göre örnek, 90-300 $\mu \mathrm{m}$ boyutlarında sslak eleme tekniği ile elendikten sonra yalnızca bileşimindeki organiklerin ayrıştırılması amacıyla $\mathrm{H}_{2} \mathrm{O}_{2}$ kimyasalı uygulanmış, tepkime sonlandıktan sonra $35^{\circ} \mathrm{C}$ 'de etüvde kurumaya bırakılmıştır. Yani numune son derece yalın bir örnek hazırlama sürecinden geçirilerek polimineral olarak hazırlanmıştır. Hazırlanan numuneler $1 \mathrm{~cm}$ çapındaki ölçüm kaplarına $10( \pm \% 20)$ miligram olacak şekilde yerleştirilmiştir.

$\mathrm{Bu}$ çalışma kapsamında tüm lüminesans ölçümleri ve 1şınlama işlemleri Riso TL/OSL-20 sistemi aracılığıyla yapılmıştır. Sistem bialkali EMI 9235QB foto çoğaltıcı tüp ve $0.120 \pm 0.05$ Gy/s doz hızına sahip ${ }^{90} \mathrm{Sr} /{ }^{90} \mathrm{Y}$ beta kaynağına sahiptir. Uyarıcı 1ş1k olarak $470 \pm 20 \mathrm{~nm}$ dalga boylu mavi 1şık ve salınan lüminesansın dedekte edilmesinde Hoya-U340 filtresi kullanılmıştır.

Eşdeğer doz ölçümlerinde Çizelge 1'de sunulan geliştirilmiş-SAR protokolü (Murray ve Wintle, 2000) kullanılmıştır. Bu teknikte numunenin artan dozlara karşı lüminesans şiddeti, hazırlanan diskin tekrarlanabilirliği ve hassasiyeti her artan doz adımında hesaba katılarak değerlendirilir.

Hesaplamalarda 32 disk kullanılarak istatistik yaklaşım yapılmıştır. İstatistiksel değerlendirmenin ilk çıktısı, disklerden elde edilen eşdeğer dozların saçılma oranıdır (Over Dispersion, OD). OD değerinin kabul edilebilir $(<\% 20)$ olması durumunda ölçümlerin tutarlı sonuç verdiği kabul edilerek örneğin yaşı (CAM, Central Age Model) ile belirlenir. Araştırılan tuzaklar çok derinde ve sıradan tuzaklara göre daha kararlı tuzaklar olduğu için sönüme dayalı lüminesans kaybı ihmal edilmiştir.

Çizelge 1. TA-OSL SAR protokolü (Şahiner vd., 2017)

Table 1. TA-OSL SAR protocol (Şahiner et al., 2017)

\begin{tabular}{|c|c|c|}
\hline Adım & Uygulama & Gözlenen \\
\hline 1 & Işınlama, $\mathrm{D}_{i}$ & \\
\hline 2 & $\mathrm{TL}, 500^{\circ} \mathrm{C}, 5^{\circ} \mathrm{C} / \mathrm{s}$ & \\
\hline 3 & $\begin{array}{c}\text { Mavi uyarım, } 200 \mathrm{~s} 180^{\circ} \mathrm{C}, \\
2^{\circ} \mathrm{C} / \mathrm{s},\end{array}$ & $\boldsymbol{L}_{i}$ \\
\hline \multirow[t]{2}{*}{4} & A ğartma & \\
\hline & Test Dozu & \\
\hline 5 & $\mathrm{TL}, 500^{\circ} \mathrm{C}, 5^{\circ} \mathrm{C} / \mathrm{s}$ & \\
\hline 6 & $\begin{array}{c}\text { Mavi uyarım, } 200 \mathrm{~s} 180^{\circ} \mathrm{C}, \\
2^{\circ} \mathrm{C} / \mathrm{s},\end{array}$ & $T_{i}$ \\
\hline 7 & A ğartma & \\
\hline 8 & İlk adıma dönüş & \\
\hline
\end{tabular}

Çizelge 1'deki protokole göre 2. adımda uygulanan TL uyarması sonucu siradan tüm lüminesans sinyalleri boşaltılır. Bu adımdan sonra sıradan tuzakların sifırlandığı oda sicaklığında mavi uyarma sonucu lüminesans salınımının izlenmesi ile kontrol edilmiştir (Şekil 4). 3. adımdaki uygun sicaklıkta OSL uyarmas sonucu salınan lüminesans sinyali ise çok derin tuzaklardan alınan sinyallerdir. SAR protokolü gereğince her artan 1şınlama döngüsünde test dozu uygulanarak hassasiyet düzeltmesi yapılarak eşdeğer doz hesaplanmıştır. 
Y1llık doz hesabında kullanılmak üzere numunenin bileşimindeki doğal radyoaktif çekirdekleri (U, Th, K, Rb) yüksek saflıkta germanyum dedektör ve kütle spektrometresi analiz teknikleriyle tespit edilmiştir (Çizelge 2). Gama spektrometresi analizleri Şahiner (2015)’te uygulanan protokole bağlı kalarak hem mutlak hem de karşılaştırmalı radyoaktivite analiz tekniklerine göre yapılmıştır. Yıllık doz hesapları Ademiec ve Aitken (1998)'te belirtilen doz dönüşüm katsayıları kullanılarak yapılmıştır.

\section{SONUÇLAR}

Şahiner 2015 ve Şahiner vd. 2017 TA-OSL tekniğiyle birkaç bin Gray (Gy) eşdeğer dozun kuvars ve polimineral örneklerde hesaplanabileceğini literatürde ilk defa göstermiştir. Ayrıca Polymeris vd. 2015a, 2015b çalışmalarında kuvars ve feldspat minerallerinin TA-OSL sinyallerine ait dozimetrik özellikler, uygun uyarım sıcaklığı ve tuzak yük yapısına ait önemli bilgiler gösterilmektedir. Bu kapsamda incelenecek numune yaşı literatürde iyi bilinen ve milyon y1l mertebesinde (1280 biny1l; Maddy vd., 2017) değerlendirilen Kula bölgesinden seçilmiştir (Şekil 2). Hazırlanan disklere Çizelge1'deki protokol uygulanarak eş değer doz hesap edilmiştir. Örneğin doğal termolüminesans (NTL) sinyali Şekil 4'de gösterilmektedir. NTL sinyalinin milyon sayım mertebesinde olduğu

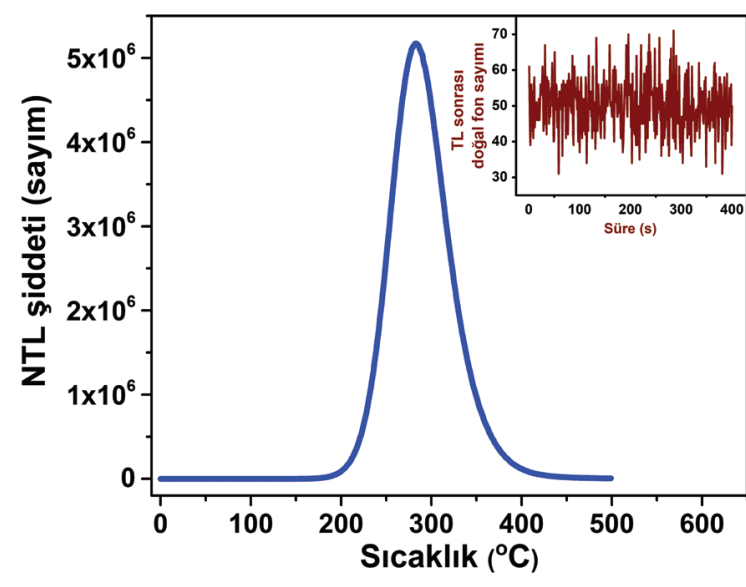

Şekil 4. Doğal TL (NTL) sinyali ve sonrasında arkaplan (background) 1şıması

Figure 4. Natural TL signal and background lamination (inset)

gözlenmektedir. $\mathrm{Bu}$ örneğin yaşlı olduğunu ve tüm tuzaklarının yeterli seviyede dolu olduğunu göstermektedir.

Doğal TL (NTL) sinyali ve fototüpün arkaplan (background) 1şıması kontrol edilmiştir. Şekil 4'de görüldügüü gibi TL sinyali oldukça parlaktır. Arkaplan 1şıması hemen TL okumasının ardından alınmıştır ve okuma sonrası fototüpte herhangi bir problem ve izotermal 1şıma gözükmemektedir. $\mathrm{Bu}$ işlem sonrasında tekrar TL ve oda sıcaklığında OSL okumalarında sinyal şiddetleri arkaplan ışıması seviyesindedir. Yani sıradan tüm tuzakların boşaltıldığg söylenebilir. 

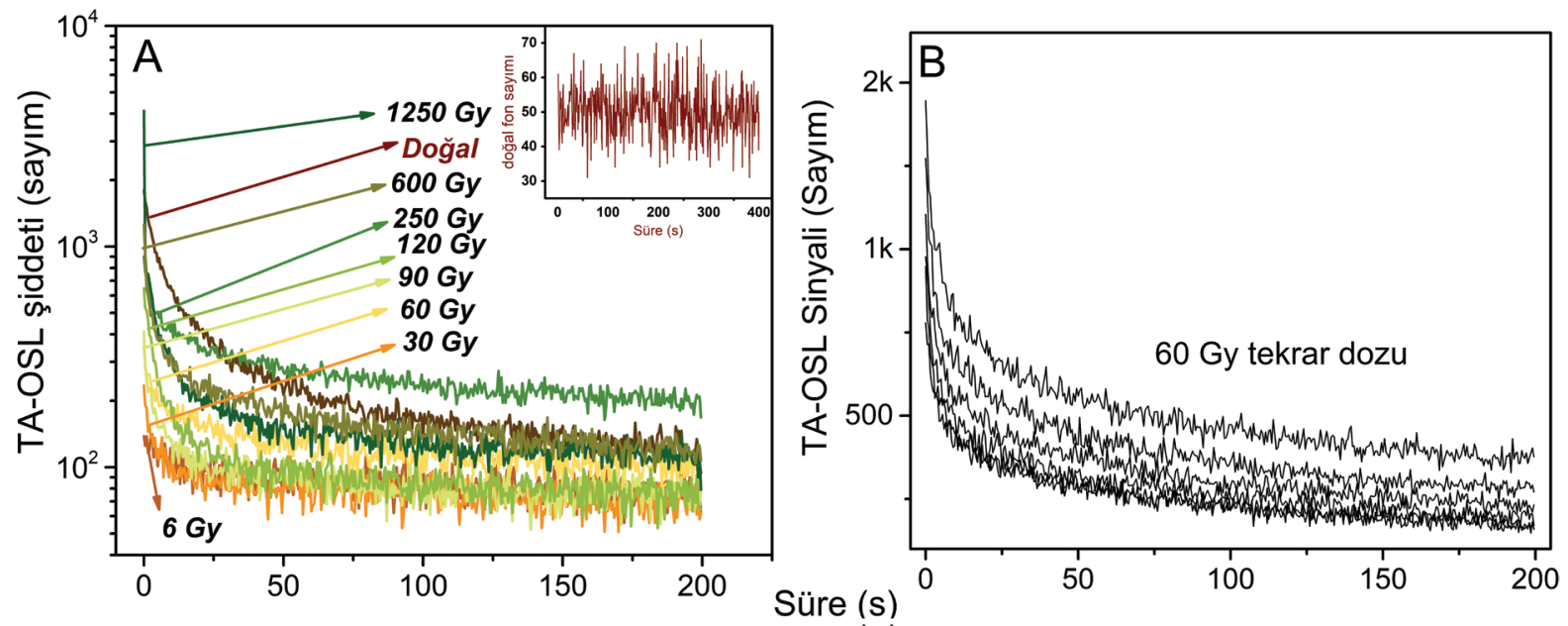

Şekil 5. Çizelge1'deki protokole göre $180^{\circ} \mathrm{C}$ 'de elde edilen TA-OSL sinyalleri (A), Artan beta dozlarına karşı TAOSL sinyalleri (B) Verilen 60 Gy beta tekrar dozuna göre TA-OSL sinyalleri

Figure 5. Obtained TA-OSL signals due to the protocol detailed in Table 1. (A), TA-OSL signals with increasing beta doses. (B) TA-OSL signals with a 60 Gy repeat beta dose.

Şekil 5A'da yapay artan ışıılama yapılmış ve doğal TA-OSL sinyallerinin yanı sıra iç şekilde arkaplan/doğal fon 1şıması (background) gösterilmektedir. Şekil 5'e göre yaklaşık 10 saniye içerisinde TA-OSL sinyalinde tuzaklarda hızlı bir boşalma olduğu anlaşılmaktadır. Ancak kuyruk kısmının da ışınlama ile birlikte arttığı gözlenmektedir. Bu durum Kütahya-Simav'dan alınmış örneklerde de gözlenmiş ve kuyruk bileşeni olarak adlandırılmıştır (Polimeris vd., 2015a, 2015b; Şahiner, 2015). TA-OSL uyarım sıcaklığı ise daha önce yapılmış çalı̧̧malardaki bulgular 1şığında $180 \quad{ }^{\circ} \mathrm{C}$ olarak seçilmiştir (Polimeris vd., 2015a, 2015b; Şahiner, 2015). TA-OSL sinyallerinin bozunum şekilleri sentetik kristaller $\left(\mathrm{Al}_{2} \mathrm{O}_{3}, \mathrm{CaF}_{2}: \mathrm{N}\right)$ ile kiyaslandığında farklı olduğu görülmektedir. Sentetik kristallerde lüminesans sinyali başlangıç bölgesinde önce artıp daha sonra azalırken incelemiş olduğumuz örnekte, siradan sürekli dalga boyu (CW-OSL) lüminesansina benzer şekilde zamanla azalan bir bozunum göstermektedir. Benzer sonuçlar Şahiner (2015) tarafindan kuvars ve polimineral numunelerde de gözlenmiş̧ir. Bu farkın nedeni doğal minerallerde safsızlıkların miktarının çok olmasından dolayı tuzaklanmış elektronların uyarıldıktan sonra iletkenlik bandına geçmeden en yakın komşuluğundaki bir merkezle lokalize yeniden birleşme yapmış olması olabilir (Şahiner, 2015).

Doz hassasiyet kontrolü için diskler aynı dozda 1şılnlanarak $180{ }^{\circ} \mathrm{C}$ sicaklıkta TA-OSL sinyalleri incelenmiştir (Şekil 5B). Ölçümlerde tekrarlanabilirlik gözlenmektedir. $\mathrm{Bu}$ durumun Şahiner, 2015 ve Polymeris vd., 2015a, 2015b çalışmasıyla çeliştiği söylenebilir. Çünkü bu çalı̧̧malarda aynı kütlede farklı disklere ait TA-OSL sinyallerinde birbiriyle uyumlu bir tekrarlanabilir sinyal gözlenememiştir.

\section{Gediz Nehri (Kula) Taraçası TA-OSL yaşı}

Gediz Nehri'nin Kula bölgesindeki yüksek taraçalarından alınan örnek içerisindeki kuvars kristallerinin çok derin tuzaklarının TA-OSL sinyali ile uyarılması, Çizelge 1'deki geliştirilmişSAR tekniği protokolü kullanılarak başarılı olarak gerçekleştirilmiştir. Şekil 6A'da numuneye ait çok derin tuzaklarından alınan TA-OSL sinyallerine bağlı doz-cevap grafiği gösterilmektedir. 
Test dozu olarak 60 Gy seçilmiştir ve eşleme işlemi lineer+eksponansiyel denklemine göre yapılmıştır. Şekilde her bir SAR döngüsünden sonra $\mathrm{T}_{\mathrm{x}} / \mathrm{T}_{\mathrm{n}}$ oranı kontrol edilerek hassasiyet değişimi düzeltilmiştir.

Çalışma kapsamında 32 disk kullanılarak ölçülen eşdeğer dozların farklı disklere göre nokta dağılımı, hata payları ile birlikte Şekil 6B'de sunulmaktadır. $\mathrm{Bu}$ grafikte ölçüm aralığının çok dışında kalarak ekstrem değerler sunan ve çok yüksek hata payına sahip $(\sim \% 40)$ iki ölçüm sonuçları kırmızı renkle gösterilmiş ve bu değerler istatistiksel değerlendirmenin dışarısında bırakılmıştır. Yaş değerlendirmesinde kullanılan 30 diskin ölçüm hata payları ortalama \%20 olarak hesaplanmıştır.

Lüminesans tarihlendirmede ölçülen eş değer doz değerlerinin istatistiksel değerlendirmesi önemli bir aşamadır. Bu değerlendirmenin ilk çıktısı, disklerden elde edilen eşdeğer dozların saçılma oranıdır (Over Dispersion, OD). OD değerinin kabul edilebilir $(<\% 20)$ olması durumunda, ölçümlerin tutarlı sonuç verdiği kabul edilerek, örneğin yaşı, merkezi yaş modeli (CAM, Central Age Model) ile belirlenir (Galbraith ve Roberts, 2012). Kula örneğinin CAM eş değer dozu ( $808 \pm 69$ Gy) olarak hesaplanmaktadır ancak
\%42 oranında yüksek saçılım gösterir. Bu yüksek saçılım oranı, ölçümlerde farklı popülasyon gruplarının değerlendirilmesinin gerekliliğini ortaya koyar (Galbraith ve Roberts, 2012). Bu amaçla sonlu karışım modeli (Finite mixture model, FMM) analizi uygulanmıştır. Bu modelin sonuçlarına ait kernel yoğunluk histogramı Şekil $6 C$ 'de, radyal dağılım grafiği de Şekil 6D'de sunulmaktadır. $\mathrm{Bu}$ analiz sonuçlarına göre $\mathrm{De}$ topluluklarından \%53'ü 1141 \pm 43 Gy; \%43'ü $582 \pm 27$ Gy değerlerinde toplulaşmaktadır. En düşük yaş modeline (MAM) göre hesaplanan De ise 242 \pm 31 Gy'dir (Çizelge 3).

Lüminesans yaş denkleminin bir diğer ana bileşeni çevresel doz hızıdır (Dr). Bu değer, örneğin konum, yükseklik ve yüzeyden derinlik bilgileri ile örneğin çevresindeki doğal radyoaktif elementlerin (U, Th, K) konsantrasyonları kullanılarak hesaplanır. Bu çalışma kapsamında, taraça çökellerindeki doğal radyoaktif elementlerin değerleri ICP-MS (ALS minerals) ve gama spektroskopisi (AÜ) teknikleriyle ölçülmüştür. Analiz sonuçları Çizelge 2'de karşılaştırılmalı olarak sunulmaktadır. Bu değerlerin, $\mathrm{U}$, Th ve $\mathrm{K}$ için sırasıyla $\sim \% 8, \% 6$ ve $\sim \% 10$ farkla birbiri içerisinde tutarlı olduğu gözlenmiştir. 


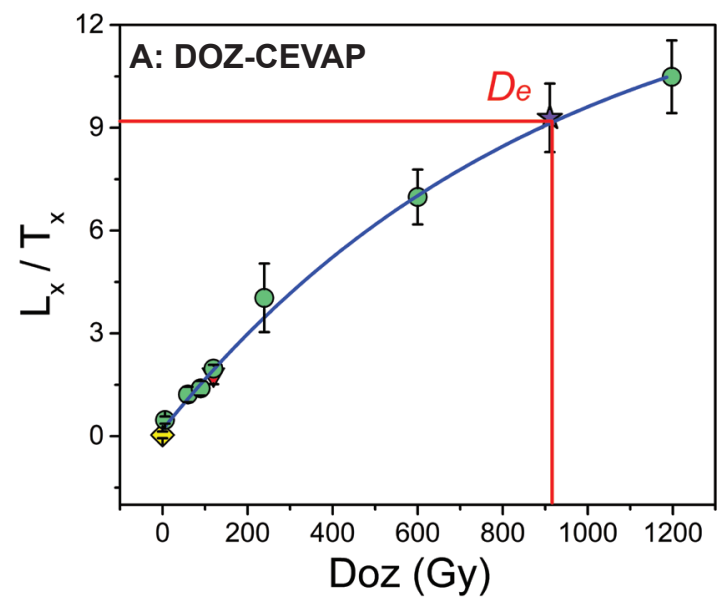

\section{C: KERNEL YOĞUNLUK}

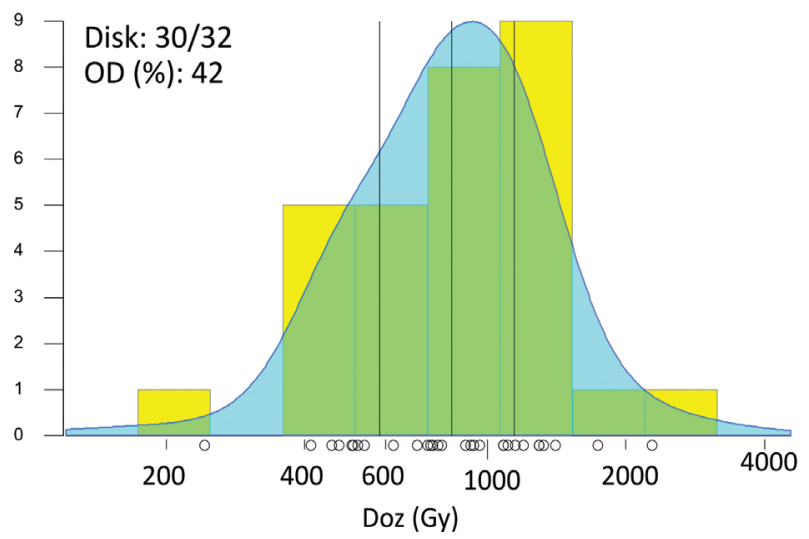

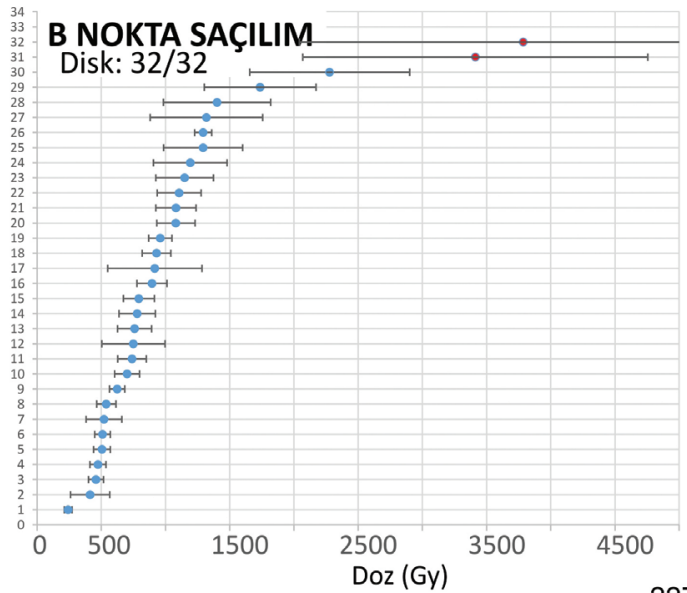

D: RADYAL

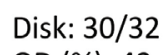

OD (\%): 42

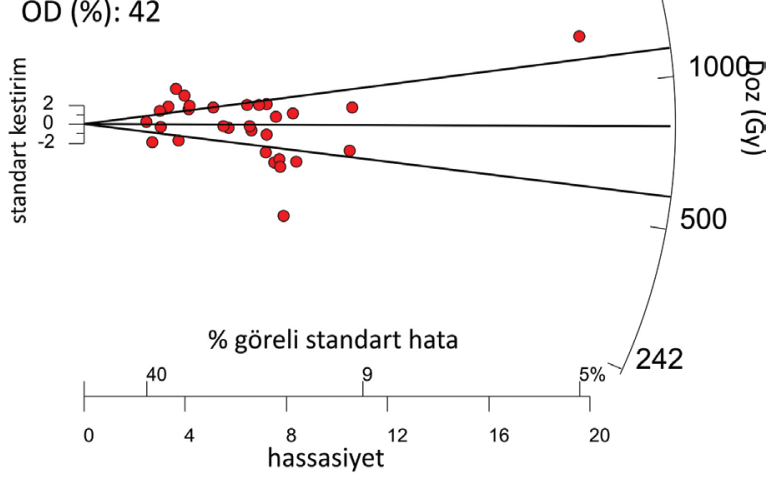

Şekil 6. Çok Derin Tuzakların TA-OSL sinyallerinin geliştirilmiş-SAR tekniğine göre eşdeğer doz değerlendirmesi (A) örnek bir diske ait doz-cevap grafiği. Ölçülen De değerlerinin, (B) nokta saçılım dağılımı (C) kernel yoğunluk dağılımı (D) Eşdeğer dozların radyal dağılımı

Figure 6. Equivalent dose evaluation of TA-OSL signal of very deep traps (A) dose response curve of sample disc (B) scatter plot (C) Kernel density histogram (D) radial plot of De values

Çizelge 2. Kula örneğinin konum ve jeokimyasal değerleri ile çevresel doz hızı

Table 2. Location and geochemical values of Kula Sample and calculated environmental dose rate

\begin{tabular}{|c|c|c|c|c|c|c|c|c|c|c|c|}
\hline 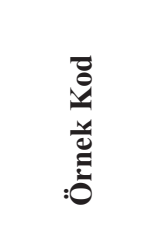 & $\begin{array}{l}\text { 音 } \\
\text { 音 }\end{array}$ & & 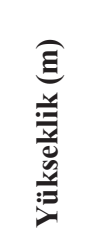 & 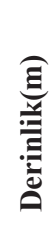 & 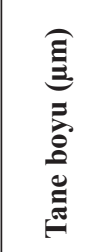 & 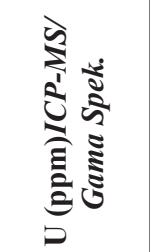 & 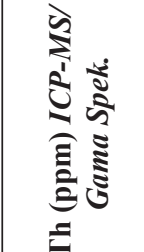 & 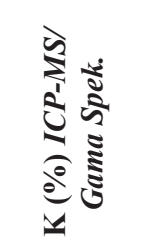 & 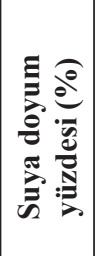 & 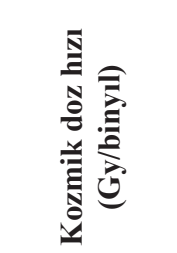 & 氛 \\
\hline \multirow[t]{2}{*}{ K1 (Kula) } & \multirow[t]{2}{*}{$30.61 \mathrm{~K}$} & \multirow[t]{2}{*}{$28.81 \mathrm{D}$} & \multirow[t]{2}{*}{542.5} & \multirow[t]{2}{*}{7.5} & \multirow[t]{2}{*}{ 90-300 } & $1.22 \pm 0.08$ & $3.70 \pm 0.14$ & $0.50 \pm 0.08$ & \multirow[t]{2}{*}{$35 \pm 5$} & \multirow[t]{2}{*}{$0.038 \pm 0.012$} & \multirow[t]{2}{*}{$1.07 \pm 0.05$} \\
\hline & & & & & & $1.33 \pm 0.04$ & $3.92 \pm 0.05$ & $0.55 \pm 0.07$ & & & \\
\hline
\end{tabular}


İstatistiksel yaklaşımla hesaplanan farklı eş değer doz değerlerinin (De), çevresel doz hızına (Dr) oranlanması ile hesaplanan yaş tablosu Çizelge 3'de sunulmaktadır.

Çizelge 3. Kula örneğinin istatistiksel analiz sonuçları ve yaş kestirimleri

Table 3. Results of the statistical analysis and age determinations

\begin{tabular}{|c|c|c|c|c|}
\hline Model & De (Gy) & $\begin{array}{c}\text { Olasiluk } \\
(\%)\end{array}$ & $\begin{array}{c}\text { Dr } \\
\text { (Gy/ } \\
\text { binyll) }\end{array}$ & $\begin{array}{c}\text { Yaş } \\
\text { (binyıl) }\end{array}$ \\
\hline CAM & $808 \pm 69$ & $*$ & \multirow{4}{*}{$1.07 \pm 0.05$} & $755 \pm 99$ \\
\hline FMM\#1 & $1141.00 \pm 43$ & 53 & & $1066 \pm 22$ \\
\hline FMM\#2 & $582 \pm 27$ & 43 & & $544 \pm 51$ \\
\hline MAM & $242 \pm 31$ & $*$ & & $226 \pm 40$ \\
\hline
\end{tabular}

\section{Gediz Nehri (Kula) Taraçası Radyometrik Yaşları}

Kula OSL örneğinin alındığı taraça seviyesi, Maddy vd., (2017) tarafindan T-1 basamağ 1 olarak ve depolanma yaşı 1280 binyıl (MIS 38) olarak önerilmiştir. $\mathrm{Bu}$ önermede, Kula'nın ilk evre volkanik faaliyetinin ürünlerinden bazaltik lav akıntısının Gediz Nehri'nin yatağını ve o dönemki taşkın ovasını örttüğü, dolayısıyla da volkanizma ile taraçanın fosilleşmesinin eş zamanlı olduğu kabul edilmiştir. Şekil 7'de Burgaz Volkanitlerinden elde edilen 28 adet radyometrik (K/Ar ve $\mathrm{Ar} / \mathrm{Ar}$ ) yaş analizinin (Richardson-Banbury, 1996; Westaway vd., 2004, 2006; Maddy vd., 2015, 2017) istatistiksel değerlendirilmesi sunulmaktadır. Buna göre lav akıntılarından elde edilen yaşların saçılım miktarı $\% 9$, ortalama değeri ise $1200 \pm 22$ binyıldır., taraçayı örten lav akıntısının radyometrik yaşların merkezi ortalaması ile taraçadan TA-OSL yöntemi kullanılarak elde ettiğimiz $1066 \pm 90$ binyıl tarihin $\% 88 \pm 10$ oranında örtüştügü görülmektedir.

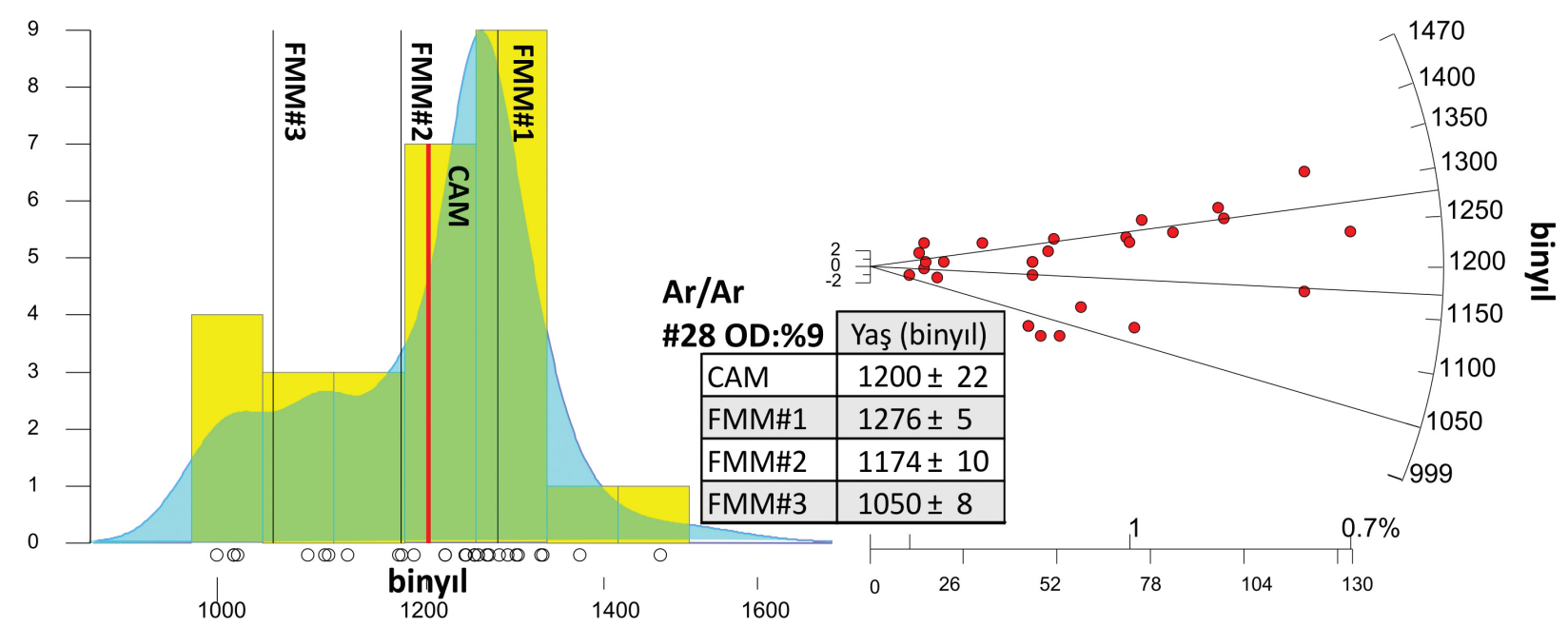

Şekil 7. OSL örneğinin alındığı taraça birimini üzerleyen Burgaz Volkanitlerine ait lavların önceki çalışmalardan derlenmiş 28 adet Ar/Ar ve K/Ar radyometrik yaş tayinin (Richardson-Banbury, 1996; Westaway vd., 2004, 2006; Maddy vd., 2015, 2017) kernel yoğunluk ve radyal diyagramlarında saçılımın çok düşük olduğu (\%9) ve ortalama yaşın $1200 \pm 22$ binyıl olduğu görülmektedir.

Figure 7. Statistical analysis of 28 radiometric dates /K/Ar and Ar/Ar) of Burgaz Volcanics (Richardson-Banbury, 1996; Westaway et al., 2004, 2006; Maddy et al., 2015, 2017). Kernel density and radial plot reveals the overdispersion is low (9\%) and the mean value is $1200 \pm 22 \mathrm{ka}$. 


\section{DEĞERLENDIRME}

Bu çalışma ile TA-OSL SAR tekniğinin potansiyeli ve doğruluğu, Kula volkanik bölgesinde yer alan Gediz Nehri'ne ait yüksek taraça seviyesinden alınan ve çökelme yaşı önceki çalışmalarda radyometrik tayinlerle sinırlanan bir örnekle sınanmıştır. $\mathrm{Bu}$ kapsamda çalışmanın önemli çıktı ve sonuçlarını aşağıdaki gibi sıralamak mümkündür.

- Yerbilimlerinde milyon yil mertebesindeki problemlere farklı yaklaşımlar getirebilen OSL tarihlendirme için yeni bir protokol önerilmiştir.

- TA-OSL tekniğiyle lüminesans dozimetri/ tarihlendirme çalışmalarda kullanılan sıradan 1sı ve 1şık ile uyarma teknikleriyle uyarılamayan çok derin tuzaklardan salınan lüminesans sinyalleri incelenmiştir.

- TA-OSL tekniği ile çok derin tuzakların kararlılığı, sönümü ve tekrarlanabilirliği incelenmiş ve lüminesans tarihlendirme çalışmalarında örnek hazırlama işlemlerinin basite indirgenebileceği gösterilmiştir.

- $\mathrm{Bu}$ tür özel tuzakların tarihlendirme çalışmalarında kullanılabilirliğinin mümkün olduğu ve yaşlı örneklere ait sıradan OSL tuzakları ile hesaplanması oldukça zor olan ait eşdeğer dozların hesaplanabileceği söylenebilir.

- TA-OSL sinyalleri için aynı diske verilen tekrar dozlarında kararlılık gözlenmiştir. $\mathrm{Bu}$ durum lüminesans tarihlendirme uygulanabilirliği açısından oldukça kritiktir.

- $\quad$ Literatürdeki diğer çalışmalara paralel olarak bu numunede de lüminesans hassasiyeti gözlenebilmiştir.

- Eşdeğer dozlarda gözlenen yüksek saçılmanın nedenlerini araştırmaya yönelik ileri çalışmalaryapılarak bu tür çok derin tuzakların lüminesans mekaniği araştırılmalıdır.
- TA-OSL tekniğinin bu çalışmadaki başarı oranı, diğer radyometrik yaş tayinlerine (Ar/ Ar) dayanan sinırlandırmaya kıyasla $\% 88 \pm 10$ olarak hesaplanmıştır.

- Bu fark henüz geliştirilmekte olan bir protokol için oldukça başarılı bir değeri ve yöntemin radyometrik yaş kontrolü bulunmayan sahalarda, kırıntılı çökel kayalarda uygulanma potansiyelini ortaya koymaktadır.

- TA-OSL tekniği benzer yaş kontrolü olan çökel paketleri üzerinde daha fazla numune, mineral tipi ve bölge üzerinde test edilmelidir.

\section{KATKI BELIRTME}

$\mathrm{Bu}$ çalışma, Türkiye Bilimsel ve Teknolojik Araştırma Kurumu (TÜBİTAK) tarafindan 117 Y208 ve 115 Y132 kodlu araştırma projeleri kapsamında desteklenmiştir.

\section{EXTENDED SUMMARY}

Research on luminescence plays a critical role on research fields such as material characterization, ionized radiation dosimetry, accident dosimetry, geochronology and detection of forgery in archaeological artifacts (McKeever, 1985; BoterJensen et al. 2003). TL (Thermoluminescence) and OSL (optically stimulated luminescence) dating techniques, which were theorized in 1970's, have improved rapidly and are used to date inorganic material. Calculating the age of the sedimentary sequences deposited during the Late Quaternary $(100-150 \mathrm{ka})$ and also the production date of archaeological artifacts (pottery, ceramics, brick, mortar etc.) is are leading applications of these techniques. Applications using protocols such as SAR (Single Aliquot Regenerative Dose; Wintle and Murray, 2000) luminescence methods can estimate radiometric ages with minimum 3-10\% error and widely accepted as a reliable dating method among radiocarbon and radioisotope techniques. 
Depending on trap-charge mechanism of crystal, natural quartz mineral's OSL signal usually compose from three regions namely fast, medium and slow components. Conventional OSL dating method focuses on the fast component (initial part of signal) of luminescence signal within the quartz crystal because it can be easily bleached by natural processes. The medium and slow components saturate higher dose limits nonetheless they suffer from bleaching problem. Therefore fast component quartz OSL dating is accepted as a reliable method among the luminescence dating society. On the other hand, the fast component part of quartz OSL tends to saturate at 100-200 $k a$ with enough bleaching with regular sun light. Making OSL dating inadequate for most longterm Quaternary geology problems. Although IRSL (InfraRed Stimulated Luminescence) signal of feldspars are particularly attractive to achieve an extended dating range compared to quartz, it has been well-know, that the potential of IRSL has not been exploited because of the loss of trapped charge with anomalous fading problem (Wintle, 1973). Recently, experiments on the physics of quartz mineral luminescence mechanism revealed a Thermal Transfer (TT) phenomena under temperatures up to 200-300 ${ }^{\circ} \mathrm{C}$. Wang et al. $(2006 a, b)$ presented that the saturation of this signal is much higher and enable to date to one million years. These signals were used to successfully used to date Chinese loess sequence with SAR protocol to $780 \mathrm{ka}$ (Wang et al., 2007) and coastal sediments at South Africa Cape coasts to $400 \mathrm{ka}$ (Jacobs et al., 2011). Another application concerns deploying violet light to stimulate deeper traps in quartz crystal lattice and successfully applied to Chinese loess sequence up to $600 \mathrm{ka}$ (Ankjoergaard et al., 2016).

Alternatively, Thermally Assisted (TA) phenomena has been introduced for higher dose estimations using deeper traps of minerals. In this study, we used Thermally Assisted (TA) optically stimulated luminescence technique (Polymeris et al., 2015a, b; Şahiner et al., 2017), a combination of TL and OSL methods to investigate the signal stored within the very deep traps (VDT) of in quartz crystal lattice which can be stimulated at higher temperatures (ie. $500{ }^{\circ} \mathrm{C}$ ), to date a sedimentary layer know to be deposited beyond the conventional OSL limit. Study of these traps has certain advantages where they are more stable, preserving luminescence signal for longer duration and have higher saturation limits.

The sample used on this research, is derived from a high terrace level deposited by Gediz River, at Kula Volcanic Field (KVF), western Turkey (Figure 1 and 2). The age of the sedimentary layer is constrained by overlying basaltic lava flow, which belongs to the first phase of volcanism in KVF. This phase (namely Burgaz Bolcanics $\beta 2$ ), is dated extensively by radiometric methods, $K / A r$ and Ar/Ar (Borsi et al., 1972; Richardson-Banbury, 1996; Westaway et al., 2003, 2004; Maddy et al., 2005, 2012a, 2012b, 2015, 2017), and appearing as a basalt plateau covering the former terrace staircases of the Gediz River. Results of recent research, combining the detailed chronology of the volcanism show that these terrace levels (11) are well correlated with Quaternary climatic cycles (MIS 52-38) where our focus terrace (T1) is attributed to MIS38 ( 1280 ka) (Maddy et al., 2005, 2017).

Figure 3 shows the detailed location (A) and measured section (B) of the OSL sample. The 3.5 $m$ long section starts with a meter thick wellrounded cobbles and boulders of the bed load (I) uncomfortably overlying the fine grained silt-clay facies of the Late Miocene Ahmetler fm. (Ersoy et al., 2010). The overlying facies is a cross bedded fine silty sand layer in $60 \mathrm{~cm}$ thickness (II) followed with $60 \mathrm{~cm}$ thick silty flood plain silts (III). The terrace section ends with a $1.2 \mathrm{~m}$ thick sheet flood alluvial silt and paleosol intercalation (IV) and covered first with basaltic ash and cobbles then the $\sim 5$ thick basaltic lava flow. The OSL sample is achieved by hammering a $20 \mathrm{~cm}$ long, $7 \mathrm{~cm}$ in 
diameter, black PVC core to the facies II. The core was tightly sealed at each end for avoiding further light exposure.

The main hypothesis of this study accepts the luminescence age of the terrace sample should be either predate closely or synchronous with the timing of volcanism. The first assumption is based on the sudden migration of the Gediz River channel with response to lava flow, and the second assumes thermal bleaching of the quartz crystals while being covered with the lava flow $\left(\sim 900^{\circ} \mathrm{C}\right)$. This hypothesis is tested using TA-OSL method.

The sample core is extracted in a safe light laboratory, wet sieved for 90-300 $\mu \mathrm{m}$ fraction and treated only with $\mathrm{H}_{2} \mathrm{O}_{2}$ to remove organic material. This separation procedure is very simple, compared with conventional OSL mineral extraction methods, and significantly reduces the laboratory time. All measurements are held with Riso TL/OSL-20 system. The system works with bialkaline EMI 9235QB photomultiplier tube and ${ }^{90} \mathrm{Sr} r{ }^{90} \mathrm{Y}$ beta source with $0.120 \pm 0.05 \mathrm{~Gy} / \mathrm{s}$ dose rate. We used $470 \pm 20 \mathrm{~nm}$ wavelength blue light source for stimulation and Hoya-U340 filter for detection of luminescence signal.

We measured equivalent doses (De) for 32 disks using the enhanced-SAR protocol (Murray and Wintle, 2000) adapted for TA-OSL technique detailed in Table 1. The TL stimulation at the second step extracts all the ordinary traps in crystal lattice, then OSL stimulation is applied and the luminescence signal is detected. We selected 60 Gy for the test dose for sensitivity correction to calculate Tc/Tn ratio at each SAR cycle. Environmental dose rate is determined by measuring natural radioactive isotopes $(U, T h, K, R b$,) with both germanium detector and ICP-MS analysis (Table 2). These twodifferent determination method is consistent for $U$, Th and $K$ concentrations with $8 \%, 6 \%$ and $10 \%$, respectively. Moreover, cosmic dose rate contribution theoretically accounts for due to geographical coordinates and depth from the surface of the sample.

The Natural Thermoluminescence (NTL) signal of a sample disc is shown in Figure 4. The NTL signal is very bright and over million counts and expressing the sample is old and all traps are adequately full. The background radiation of the phototube is measured right after the TL measurement and no saturation problem or isothermal radiation is detected. Figure $5 A$ shows overlapping TA-OSL signal after exposing the disks with increasing artificial doses. The signals show a fast discharge in 10 seconds but a buildup in the tail section with increasing exposure to artificial radiation. This phenomenon was also reported by Polymeris et al. (2015 a, b) and Shahiner (2015) and named as the tail component.

Figure 6 summarizes the results of TASAR protocol, where A shows the dose response curve of a sample disc, fitting is calculated using lineer+exponential equation. B shows the radial plot distribution, $C$ shows the kernel density histogram and $D$ is the scatter plot drawn by the De values of the all 32 discs. The error rates of the measurements is high by mean of $20 \%$ also reported in previous studies (Şahiner, 2015). The statistical evaluation after dismissing the extreme measurements with high error values reveals a high overdispersion value (42\%) with a CAM value of $(808 \pm 69 \mathrm{~Gy})$. This high dispersion value requires statistical approach in order to determine the age of the sample (Galbraith and Roberts, 2012). We employed finite mixture model (FMM) to calculate dose populations where $53 \%$ of the measurements populate at $1141 \pm 43$ Gy and $43 \%$ $582 \pm 27$ Gy, the minimum age model (MAM) shows $242 \pm 31$ Gy (Table 3).

We evaluated 28 radiometric ages retrieved from the Burgaz Volcanic phase of KVP (Figure 7). The central age of these ages is $1200 \pm 22 \mathrm{ka}$ with dispersion value as $9 \%$. This age coincides $(88 \pm 10 \%)$ with the TA-OSL age $(1066 \pm 90 \mathrm{ka})$ 
showing a promising success of a developing technique.

\section{ORCID}

Eren Şahiner (D) https://orcid.org/0000-0002-7159-2491

M. Korhan Erturaç D https://orcid.org/0000-0002-6501-760X

Niyazi Meriç D https://orcid.org/0000-0003-1562-1312

\section{DEĞINILEN BELGELER}

Adamiec, G., and Aitken, M. J., 1998. Dose-rate conversion factors: update. Ancient TL, 16, 2, 3750 .

Aitken, M. J.,1985. Thermoluminescence dating. Academic press, Orlando, Florida.

Ankjærgaard, C., Guralnik, B., Buylaert, J. P., Reimann, T., Yi, S. W., \& Wallinga, J., 2016. Violet stimulated luminescence dating of quartz from Luochuan (Chinese loess plateau): Agreement with independent chronology up to $600 \mathrm{ka}$. Quaternary Geochronology, 34, 33-46.

Ankjærgaard, C., Guralnik, B., Porat, N., Heimann, A., Jain, M., \& Wallinga, J., 2015. Violet stimulated luminescence: geo-or thermochronometer?. Radiation Measurements, 81, 78-84.

Ankjærgaard, C., Jain, M., and Wallinga, J., 2013. Towards dating Quaternary sediments using the quartz Violet Stimulated Luminescence (VSL) signal.Quaternary Geochronology, 18, 99-109.

Borsi, M., Ferrara, G., Innocenti, F., Mazzuoli, R., 1972. Geochoronology and petrology of recent volcanics of Eastern Aegean Sea, Bull. Volcan., 36/1, 473-496.

Bøtter-Jensen, L., McKeever, S. W., Wintle, A. G., 2003. Optically stimulated luminescence dosimetry. Elsevier.

Bulur, E., Göksu, H.Y. 1999. Phototransferred thermoluminescence from a-Al2O3:C using blue light emitting diodes. Radiation Measurements 30, 203-206.

Ercan, T., 1993. Interpretation of geochemical, radiometric and isotopic data on Kula volcanics (Manisa-western Anatolia). Geol. Bull. Turk. 36 (1), 113-129.
Ercan, E., Türkecan, A., Dinçel, A., Günay, E., 1983. Kula-Selendi (Manisa) Bölgesinin Jeolojisi. Jeoloji Mühendisligi 17, 3-28.

Ersoy, Y., Helvac1, C., Sözbilir, H., 2010. Tectonostratigraphic evolution of the NEeSW-trending superimposed Selendi basin: implications for late Cenozoic crustal extension in Western Anatolia, Turkey. Tectonophysics 488, 210-232.

Erinç, S., 1970. Kula-Adala arasında genç volkan röliyefi.İ.Ü. Coğrafya Ens. Derg. 17, 148-167.

Galbraith, R.F. and Roberts R.G., 2012. Statistical aspects of equivalent dose and error calculation and display in OSL dating: An overview and some recommendations. Quaternary Geochronology, 11, 1-27.

Gorp, W., Veldkamp, A., Temme, A.J.A.M., Maddy D., Demir T., van der Schriek T., Reimannf, T., Wallinga J., Wijbrans J., Schoorl, J.M. 2013. Fluvial response to Holocene volcanic damming and breaching in the Gediz and Geren Rivers, western Turkey, Geomorphology 201, 430-448.

Heineke, C., Niedermann, S., Hetzel, R., \& Akal, C. (2016). Surface exposure dating of Holocene basalt flows and cinder cones in the Kula volcanic field (Western Turkey) using cosmogenic $3 \mathrm{He}$ and $10 \mathrm{Be}$. Quaternary Geochronology, 34, 81-91.

Jacobs, Z., Roberts, RG., Lachlan, TJ., Karkanas, P., Marean, CW., Robert,s DL., 2011. Development of the SAR TT-OSL procedure for dating Middle Pleistocence dune and shallow marine deposits along the southern Cape coast of South Africa. Quat Geochronology 6:491-513.

Kitis, G., Kiyak, N.G., Polymeris, G.S. and Pagonis, V. 2010. Investigation of OSL signals from very deep traps in unfired and fired quartz samples. Nuclear Instruments and Methods in Physics Research B 268, 592-598.

Kitis, G., Polymeris, G.S., Pagonis, V. and Tsirliganis, N.C. 2013. Anomalous Fading of OSL signals originating from very deep traps in Durango apatite. Radiation Measurements 49 73-81.

Kittel, C. 2004. Introduction to Solid State Physics, 8th Edn, John Wiley and Sons, Ltd. 675p.

Maddy, D., Demir, T., Bridgland, D., Veldkamp, A., Stemerdink, C., van der Schriek, T., Westaway, R., 2005. An obliquity-controlled Early Pleistocene river terrace record from Western Turkey? Quat. Res. 63, 339-346. 
Maddy, D., Demir, T., Veldkamp, A., Bridgland, D.R., Stemerdink, C., van der Schriek, T., Schreve, D., 2012. The obliquity-controlled early Pleistocene terrace sequence of the Gediz River, Western Turkey: a revised correlation and chronology. J. Geol. Soc. Lond. 169, 67-82.

Maddy, D., Schreve, D., Demir, T., Veldkamp, A., Wijbrans, J.R., van Gorp, W., van Hinsbergen, D.J.J., Dekkers, M.J., Scaife, R., Schoorl, J.M., Stemerdink, C., van der Schriek, T., 2015. The earliest securely-dated hominin artefact in Anatolia? Quat. Sci. Rev. 109, 68-75.

Maddy, D., Veldkamp, A., Demir, T., van Gorp, W., Wijbrans, J.R., van Hinsbergen, D.J.J., Dekkers, M.J., Scahreve, D., Schoorl, J.M., Scaife, R., Stemerdink, C., van der Schriek, T., Bridgland, D.R., Aytaç, A.S., 2017. The Gediz River fluvial archive: A benchmark for Quaternary research in Western Anatolia. Quat. Sci. Rev. 166, 68-75.

McKeever, S. W. 1988. Thermoluminescence of solids (Vol. 3). Cambridge University Press.

Murray, A. S., and Wintle, A. G. 2000. Luminescence dating of quartz using an improved singlealiquot regenerative-dose protocol. Radiation measurements, 32(1), 57-73.

Ozaner, F.S., 1992. Detecting the polycyclic drainage evolution in Kula region (western Turkey) using aerial photographs. ITC J. 1992-1993, 249-253.

Polymeris, G.S. and Kitis, G. 2012. Thermally assisted photo transfer OSL from deep traps in A12O3:C grains exhibiting different TL peak shapes. Applied Radiation and Isotopes, 70(10), 2478-2487.

Polymeris, G.S., Raptis, S., Afouxenidis, D., Tsirliganis, N.C. and Kitis, G. 2010. Thermally assisted OSL from deep traps in Al2O3:C. Radiation Measurements 45, 519-522.

Polymeris, G.S., Şahiner, E., Meriç, N. and Kitis, G. 2015a. Experimental features of natural thermally assisted OSL (NTA-OSL) signal in various quartz samples; preliminary results. Nuclear Instruments and Methods in Physics Research Section B: Beam Interactions with Materials and Atoms. 349, 24-30.

Polymeris, G.S., Şahiner, E., Meriç, N. and Kitis, G. 2015b. Thermal assistance in TA - OSL signals of feldspar and polymineral samples; comparison with the case of pure quartz. Radiation Measurements. 81, 270-274.
Richardson-Bunbury, J.M., 1996. The Kula volcanic field, western Turkey: the development of a Holocene alkali basalt province and the adjacent normal faulting graben. Geol. Mag. 133, 275-283.

Şahiner, E., 2015. Paleosismolojik çalışmalarda TL/ OSL ve ESR yöntemlerinin kullanılması: KütahyaSimav ve Kuzey Anadolu fay hatt1. Doktora tezi. Ankara Üniversitesi Fen Bilimleri Enstitüsü Fizik Mühendisliği Anabilim Dalı. 216s.

Şahiner, E., Meriç, N., \& Polymeris, G. S. (2017). Thermally assisted OSL application for equivalent dose estimation; comparison of multiple equivalent dose values as well as saturation levels determined by luminescence and ESR techniques for a sedimentary sample collected from a fault gouge. Nuclear Instruments and Methods in Physics Research Section B: Beam Interactions with Materials and Atoms, 392, 21-30.

Şen, E., Erturaç, M.K., Gümüş, E. 2017. Quaternary monogenetic volcanoes scattered on a horst: the bountiful landscape of Kula. In: Landscapes and Landforms of Turkey. Chapter:34, Ed. Kuzucuoglu, C., Çiner, A., Kazanc1, N. Springer.

Veldkamp, A., Candy, I., Jongmans, A.G., Maddy, D., Demir, T., Schoorl, J.M., Schreve, D., Stemerdink, C., van der Schriek, T., 2015. Reconstructing Early Pleistocene $(1.3 \mathrm{Ma})$ terrestrial environmental change in western Anatolia: did it drive fluvial terrace formation? Palaeogeogr. Palaeoclimatol. Palaeoecol. 417, 91-104.

Wang, W-D., Xia, J-D., Zhou, Z-X., 2006a Thermoluminescence dating of the ancient Chinese porcelain using a regression method of saturation exponential in pre-dose technique. Sci China Ser E: Technol Sci 49:194-209

Wang, XL., Lu, YC., Wintle, AG., 2006b. Recuperated OSL dating of fine-grained quartz in Chinese loess. Quat Geochronol 1:89-100

Wang, XL., Wintle, AG., Lu, YC., 2007 Testing a single-aliquot protocol for recuperated OSL dating. - Radiation Measurements 42:380-391

Westaway, R., Pringle, M., Yurtmen, S., Demir, T., Bridgland, D.R., Rowbottom, G., Maddy, D., 2004. Pliocene and Quaternary regional uplift in western Turkey: the Gediz river terrace staircase and the volcanism at Kula. Tectonophysics 391, 121-169.

Westaway, R., Guillou, H., Yurtmen, S., Beck, A., Bridgland, D.R., Demir, T., Rowbottam, G., 2006. 
Late Cenozoic uplift of western Turkey: improved dating and numerical modelling of the Gediz river terrace staircase and the Kula Quaternary volcanic field. Glob. Planet. Change 51, 131-171.
Wintle, AG., 1973. Anomalous fading of thermoluminescence in mineral samples. Nature 245: $143-144$ 